\title{
LA EXPANSIÓN DE LAS ESPECIES DE PLUCHEA CASS. (ASTERACEAE) Y SU POTENCIAL COMPORTAMIENTO INVASOR EN TENERIFE (ISLAS CANARIAS)
}

\author{
María Leticia Rodríguez Navarro*, Beatriz Fariña Trujillo*, \\ Atteneri Rivero Quintero ${ }^{* *}$
}

\section{RESUMEN}

Se aborda el potencial comportamiento invasor de las especies del género Pluchea en la isla de Tenerife, mediante su caracterización corológica, implementada con datos propios, de su Cabildo Insular, del equipo de alerta temprana del Gobierno de Canarias (REDEXOS) y la información inédita cedida por otros investigadores. Se recomienda su incorporación al Catálogo español de especies exóticas invasoras, así como en el futuro Catálogo regional. Por último, se aportan recomendaciones para su manejo y control.

Palabras Clave: especies potencialmente invasoras, distribución, Pluchea, control, Tenerife.

THE EXPANSION OF PLUCHEA CASS.

(ASTERACEAE) SPECIE AND ITS POTENTIAL INVASIVE

BEHAVIOUR IN TENERIFE (CANARY ISLANDS)

\section{Abstract}

The invasive potential behaviour of the species of the genus Pluchea on the island of Tenerife, through its chorological characterization, realized with data recorded by the authors, from the Island Council, the early warning team of the Canary Islands Government (REDEXOS) and unpublished information released by other researchers. From this analysis, its incorporation in the Spanish Catalogue of invasive alien species is recommended and, also, in the future regional Catalogue. Finally, we provide recommendations related to its management and control.

Keywords: potentially invasive species, distribution, Pluchea, control, Tenerife. 


\section{INTRODUCCIÓN}

Pluchea Cass. es un género de la familia Asteraceae que se distribuye exclusivamente por regiones tropicales y subtropicales del Viejo y el Nuevo Mundo en ambos hemisferios (King-Jones 2001). Aparece en Asia, norte y sur de América, África y Australia (Qaiser \& Abid 2003) y cuenta con 45-80 especies (King-Jones 2001; Oliver i Martínez-Fornés et al. 2007). Salvo algunas excepciones, son arbustos y subarbustos adaptados al severo estrés propio de los ámbitos donde se distribuye: altas temperaturas, bajas precipitaciones, estaciones secas y sequías prolongadas (King-Jones 2001).

En la isla de Tenerife se han naturalizado dos especies de este género: $P$. ovalis (Pers.) DC., cuya distribución abarca del oeste de África a la península de Arabia (King-Jones 1999), y P. dioscoridis (L.) DC. está muy extendida en África tropical y subtropical y la península arábiga, apareciendo también en Australia, exclusivamente en Queensland (King-Jones 2001).

Ambas especies comparten distribución en Angola, Burundi, Tanzania, Chad, Etiopía, Kenia, Sudán, Eritrea, Somalia, Uganda, Omán, Arabia Saudí, Qatar, Emiratos y Bahrain (http://www.plantsoftheworldonline.org), e incluso pueden encontrarse creciendo en las mismas áreas en los países de Burundi y Tanzania (King-Jones, 2001).

En Tenerife $P$. ovalis se detectó en el año 2000 en el casco urbano del municipio de Adeje (R. Mesa, com. pers.), posteriormente Padrón-Mederos et al. (2009) recolectan material en el borde de carretera de la variante La Caleta-Golf de Adeje, en el barranco del Agua. Verloove \& Reyes-Betancort (2011) confirman la rápida propagación por numerosas localidades entre los núcleos poblacionales de Adeje y Playa de Las Américas, y Barone \& Hernández (2014) amplían la corología a los municipios cercanos de Arona, Santiago del Teide y Guía de Isora sin rebasar los $200 \mathrm{~m}$ de cota máxima.

Martín-Osorio \& Wildpret (2014) encuentran P. dioscoridis (L.) DC. en el interior de una presa artesanal que recoge las aguas pluviales estacionales en el complejo de San Blas, municipio de San Miguel de Abona.

En su zona de procedencia, $P$. ovalis aparece en suelos con humedad edáfica relativamente alta, pastizales y, ocasionalmente, en terrenos pantanosos, en márgenes de ríos, arroyos, acequias y humedales temporales o permanentes no muy salados (King-Jones 1999; Charco 2001) y, P. dioscoridis en zonas donde el agua subterránea está cerca de la superficie, o cerca de bancos de agua, caminos y pantanos (King-Jones 1999; Shaltout \& Slima 2007).

* Técnica del Servicio de Impacto Ambiental de la Viceconsejería de Lucha contra el Cambio Climático del Gobierno de Canarias. Santa Cruz de Tenerife, Islas Canarias, España. Autor para la correspondencia: 1rodnav@gobiernodecanarias.org.

** Licenciada en Biología. Santa Cruz de Tenerife, Islas Canarias, España.

*** Licenciada en Biología. Santa Cruz de Tenerife, Islas Canarias, España. 
Quizás la distribución de las especies del género Pluchea pudiera estar vinculada a su uso medicinal. En África, Arabia e India, hojas y tallos se emplean como purgante, antipirético o para tratar inflamaciones o forúnculos dolorosos (King-Jones 2001). Con la decocción de hojas frescas de $P$. dioscoridis se trata la epilepsia en niños, los cólicos, como carminativo y como remedio para el enfriamiento; además, en Tanzania, la decocción de la corteza de la raíz se emplea contra la esterilidad en mujeres e impotencia en hombres (Shaltout \& Slima 2007). El extracto etanólico de las raíces de $P$. ovalis sirve en el tratamiento del asma (Oliver i Martínez-Fornés et al. 2007).

El comportamiento invasor de $P$. dioscoridis y $P$. ovalis en diferentes lugares de África e incluso para Tenerife se recoge en varias bases de datos (Global Register of Invasive Species y Global Biodiversity Information Facility) y literatura (Simpson 1932; Shaltout \& Slima 2007).

En atención a la peligrosidad de varias especies exóticas invasoras en la isla de Tenerife, el Área de Desarrollo Sostenible y Lucha contra el Cambio Climático de su Cabildo Insular y el Gobierno de Canarias trabajan en su control, estableciendo prioridades en las intervenciones en función del grado de peligrosidad y de los valores ambientales afectados. Al mismo tiempo, abren líneas de cooperación con el Centro Superior de Investigaciones Científicas (CSIC) para el ensayo de diferentes métodos de actuación en las más problemáticas (Elejabeitia \& Delgado 2018).

Aunque los taxones aquí tratados no están incluidos en el Catálogo español de especies exóticas invasoras -Real Decreto 630/2013, de 2 de agosto-, las citadas administraciones públicas están procediendo al control de $P$. ovalis en aplicación del artículo 9 del citado Real Decreto 630/2013 al «... constatarse la existencia de una amenaza grave producida por la aparición de una especie exótica invasora, incluida o no en el catálogo...».

En 2017, con financiación europea, se dio inicio al Proyecto de Control de Flora Exótica Invasora en Tenerife, que ha continuado en años posteriores realizando trabajos de control. Desde ese mismo año el equipo del proyecto REDEXOS del Gobierno de Canarias incluye, por su carácter invasor y como respuesta temprana, el control de $P$. ovalis especialmente en espacios naturales protegidos y su entorno inmediato.

Respecto a los objetivos del trabajo se plantean: identificar las posibles vías de introducción y dispersión; recopilar cartográficamente la distribución de ambas especies en la isla de Tenerife y, en concreto, con respecto a los espacios naturales protegidos de la Red Canaria y de la Red Natura 2000; y reunir las prácticas de manejo y control que se están aplicando con estas especies.

\section{MATERIAL Y MÉTODOS}

Se ha realizado una exhaustiva búsqueda bibliográfica sobre las dos especies tras consulta al Banco del Inventario Natural de Canarias (https://www.biodiversidadcanarias.es), se analizan las citas y la documentación referida a las mismas, se buscó información en floras básicas y publicaciones específicas, se tomaron en con- 
sideración los datos corológicos aportados por el Cabildo Insular de Tenerife y por REDEXOS, además de los cedidos por R. Barone y F. Hernández, y los obtenidos en el trabajo de campo del presente estudio y, por último, las comunicaciones personales de diversos interlocutores.

Entre los meses de septiembre y noviembre de 2019 se recolectaron muestras, tomando datos sobre la fenología (tamaños de edad y estado reproductivo), corología (incluyendo la altitud) y georreferenciación mediante el empleo de un dispositivo GPS marca Garmin modelo Etrex Vista Hcx. Igualmente, se han fotografiado detalles morfológicos de los ejemplares y de su entorno. Adicionalmente, se realizaron visitas a los principales campos de golf presentes en la isla de Tenerife: campo de golf Los Palos, Amarilla Golf, San Andrés Golf del Sur, Golf Costa Adeje, Abama Golf, Buenavista Golf y Real Club de Golf de Tenerife. El Golf Las Américas no otorgó autorización para acceder a sus instalaciones; no obstante, se visitaron sus exteriores.

El material recolectado, tras su prensado y determinación, fue depositado en el Herbario ORT del Jardín de Aclimatación de La Orotava, sito en el Puerto de la Cruz, para su registro.

Unificando las coordenadas UTM (-WGS 84-) de los datos corológicos de las especies de Pluchea estudiadas provenientes del Cabildo Insular, REDEXOS, R. Barone y F. Hernández y propios, se han representado en mapas, reflejando igualmente la Red de Espacios Naturales Protegidos de Canarias, mediante el uso de herramientas SIG (ArcGIS 10.5.1) y usando como base cartográfica la extraída de los servicios cartográficos básicos aportados por el Sistema de Información Territorial de Canarias-IDECanarias (https://www.idecanarias.es).

La similitud morfológica entre ambas especies (Chevalier 1935) y la variabilidad de P. ovalis (King-Jones 1999) dificultan la identificación. Para la determinación del material recolectado seguimos el criterio adoptado por King-Jones (1999), según la siguiente dicotomía:

- hojas ampliamente auriculadas y/o decurrentes, indumento grisáceo lanoso......... P. ovalis - hojas ligeramente auriculadas, nunca decurrentes, indumento piloso .............P. dioscoridis

\section{RESULTADOS}

Fruto de las visitas de campo se recolectaron un total de 18 pliegos (ver anexo I) que tras su identificación fueron registrados y depositados en el Herbario ORT. De ellos 9 corresponden a $P$. ovalis y, el resto, a $P$. dioscoridis (ver figuras 1 y 2 ).

Reseñar que, en la charca de la Reserva Ambiental San Blas, locus donde Martín-Osorio \& Wildpret (2014) citan P. dioscoridis, hemos herborizado una muestra asimilable a $P$. ovalis al presentar hojas decurrentes en la base, al menos las inferiores y anchamente auriculadas. Las plantas vistas en campo presentan fauna invertebrada asociada, por lo que pudiera ser una especie hospedadora de parásitos y/o patógenos. En concreto, se detectaron individuos del género Bemisia (mosca blanca), familia Aleyrodidae. 

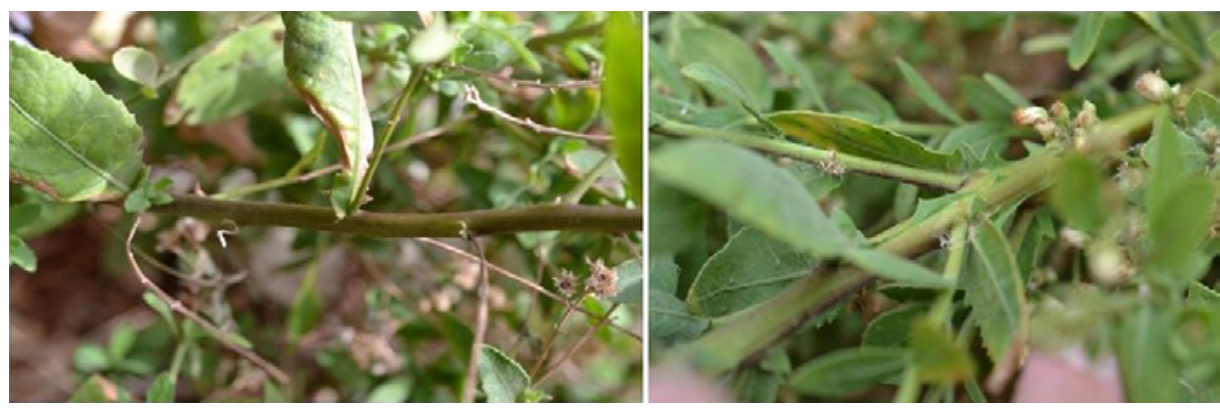

Figura 1. En la imagen de la izquierda Pluchea dioscoridis (L.) DC., y en la de la derecha P. ovalis (Pers.) DC. con hojas auriculadas y decurrentes.
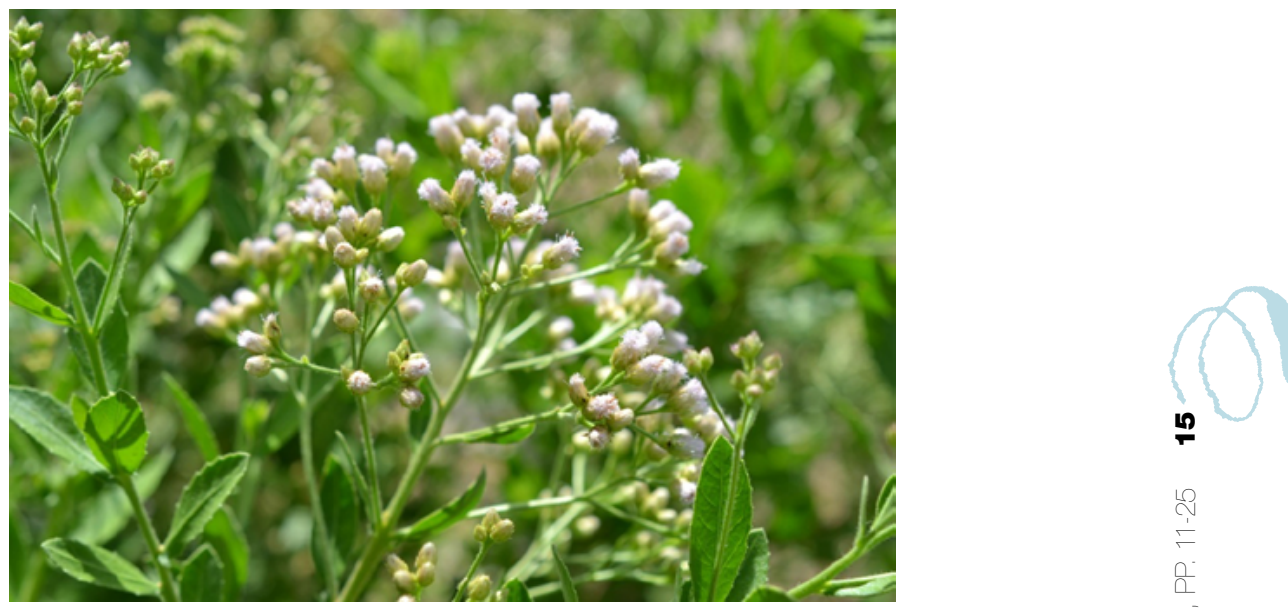

Figura 2. Planta de $P$. dioscoridis en flor en el mes de octubre.

De los mapas obtenidos (ver figuras 3 y 4 ) con datos de diversa procedencia (-Cabildo Insular, REDEXOS, R. Barone y F. Hernández, que está referida a $P$. ovalis- y los herborizados en campo $-P$. ovalis y $P$. dioscoridis -$)$ se infiere que las especies del género Pluchea presentes en Tenerife, principalmente aparecen en entornos antropizados, ocupando una ancha banda entre los $0 \mathrm{~m}$ s.n.m. como punto más bajo y los casi $900 \mathrm{~m}$ de altitud en Santiago del Teide, y concentradas desde el municipio de Granadilla de Abona hasta el de Buenavista. La localidad más oriental sujeta a control fue en un parterre de la mediana de la carretera TF-29, a escasos $500 \mathrm{~m}$ de la autopista TF-5, principal corredor viario que une la conurbanación Santa Cruz-La Laguna. 


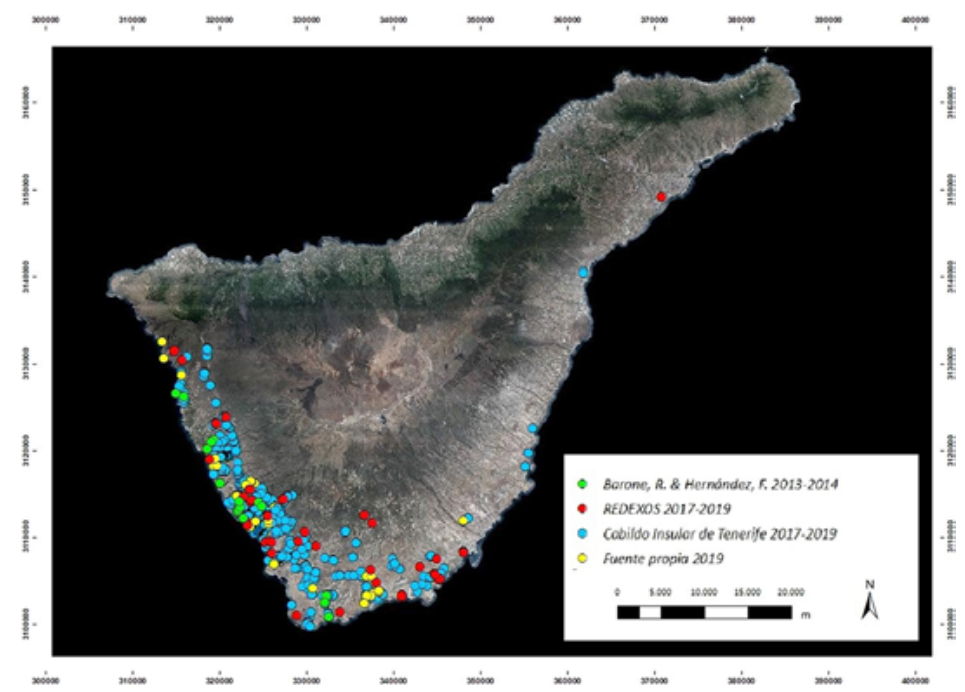

Figura 3. Representación de los datos de las especies del género Pluchea presentes en la isla de Tenerife según su procedencia: Cabildo Insular de Tenerife, REDEXOS, información inédita cedida por investigadores y el material herborizado (base cartográfica extraída de los servicios cartográficos básicos aportados por el Sistema de Información Territorial de Canarias-IDECanarias -https://www.idecanarias.es-).

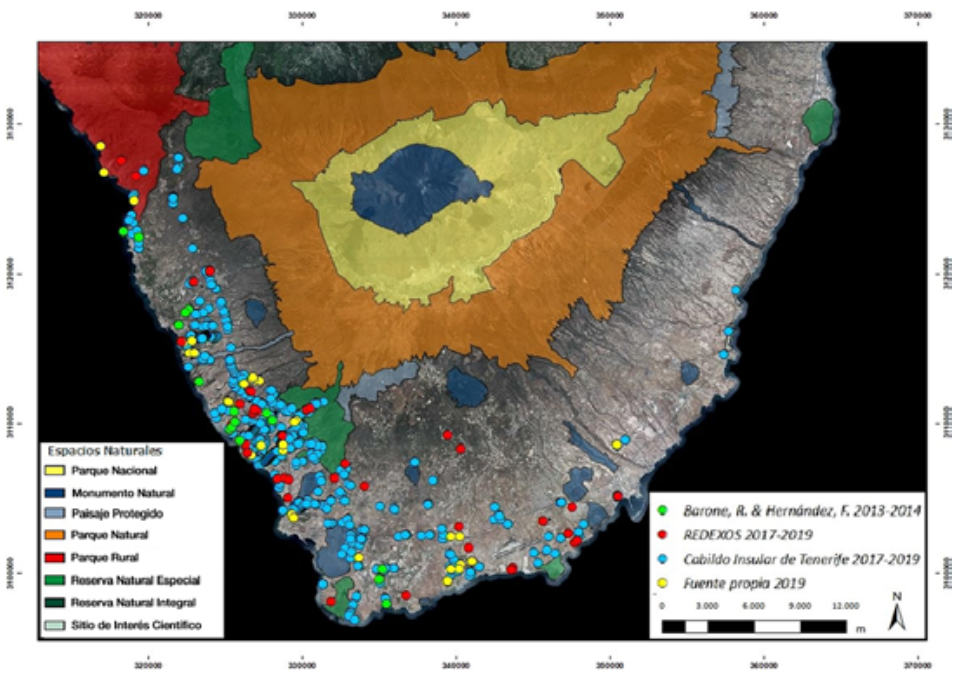

Figura 4. Representación de los datos de las especies del género Pluchea presentes en la isla de Tenerife de diversa procedencia, respecto a los espacios naturales protegidos de la Red Canaria existentes en dicha isla (base cartográfica extraída de los servicios cartográficos básicos aportados por el Sistema de Información Territorial de Canarias-IDECanarias -https://www.idecanarias.es-). 


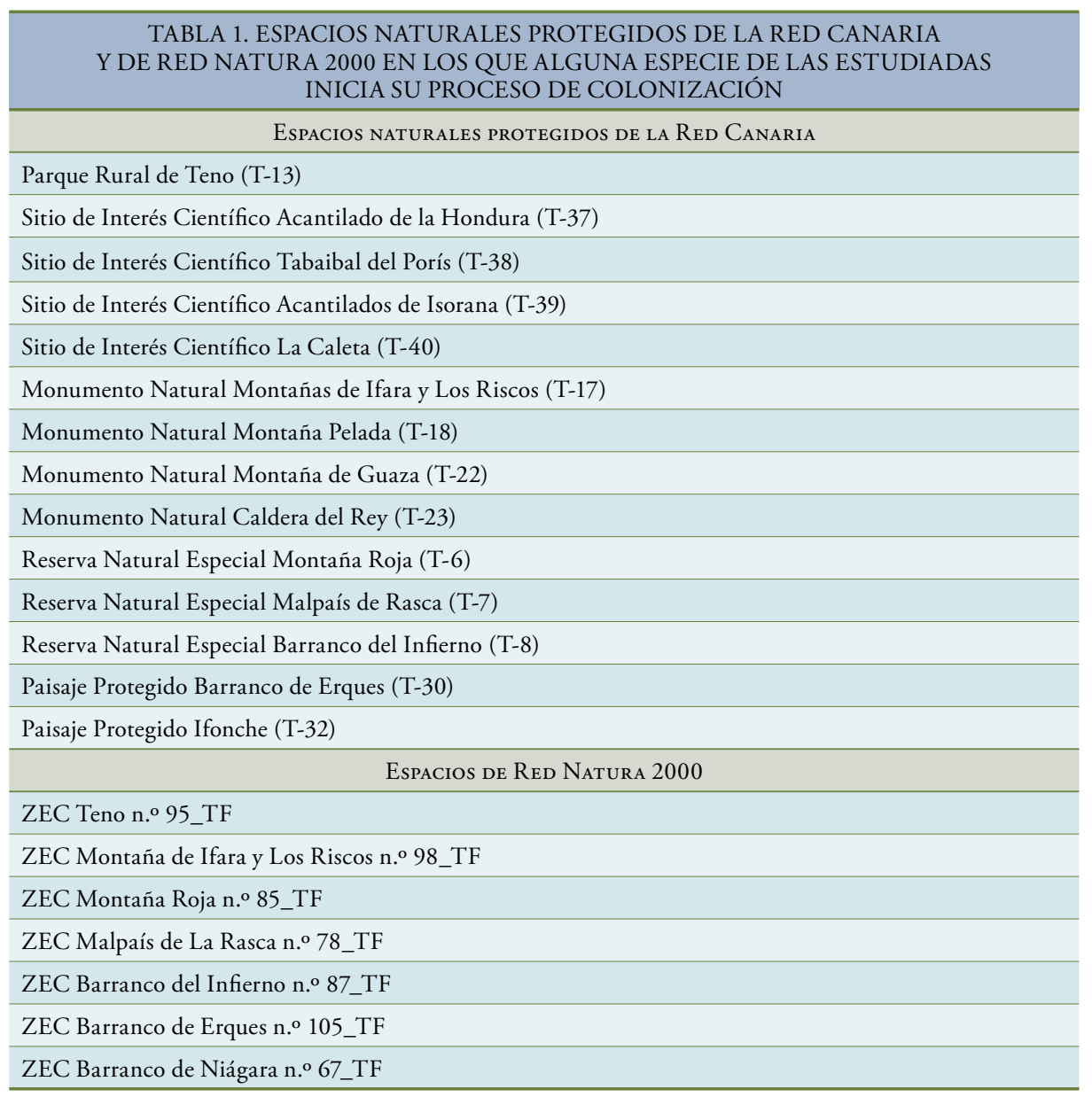

Señalar que $P$. ovalis ha alcanzado o se encuentra en la cercanía de espacios naturales protegidos de la Red Canaria y de la Red Natura 2000 (ver tabla 1), lo que supone un impacto directo sobre el $16 \%$ de los espacios naturales protegidos presentes en la isla y amenazando a la periferia del $21 \%$ (ver figuras 5 y 6 ). Hay individuos de una de estas dos especies en los siguientes hábitats naturales de interés comunitario: 5330 «Matorrales termomediterráneos y pre-estépicos», 1250 «Acantilados con vegetación de las costas macaronésicas (flora endémica de estas costas)» $\mathrm{y}$ 92D0 «Galerías ribereñas termomediterráneas (Nerio-Tamaricetea) y del sudoeste de la península ibérica (Securinegion tinctoriae)».

Respecto al manejo y control, las cuadrillas de control trasladan que han aplicado los siguientes métodos: tala, desenraizado, aplicación de herbicida, pudrición de los troncos y quema controlada. En los ejemplares más jóvenes funciona el arranque manual, y en los de mayor porte el tratamiento con herbicida en tocones 

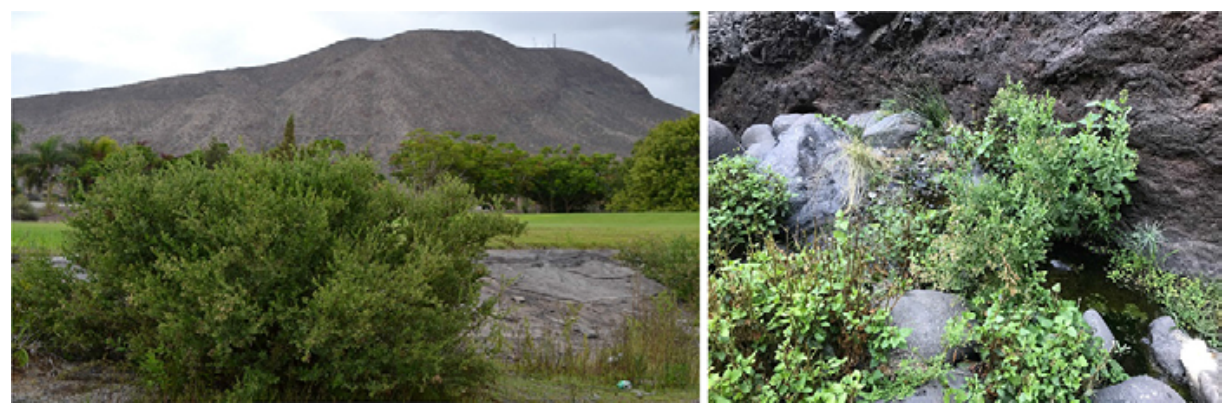

Figura 5. En la imagen de la izquierda, individuo de $P$. ovalis en el campo de golf Los Palos, a menos de $400 \mathrm{~m}$ de los límites del Monumento Natural de Montaña Guaza (T-22).

En la de la derecha, la misma especie en el cauce del barranco de Masca, dentro del Parque Rural de Teno y su ZEC homónimo.

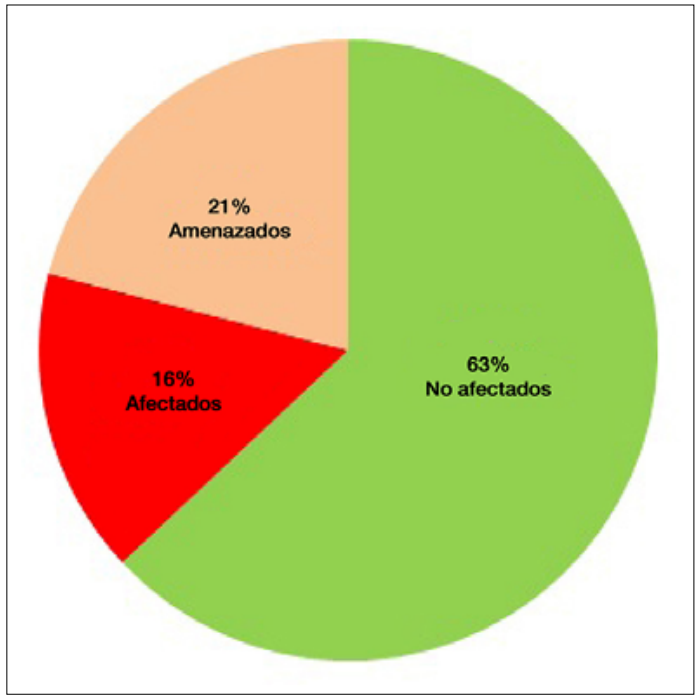

Figura 6. Porcentajes de los espacios naturales protegidos de la Red Canaria afectados y amenazados por las especies del género Pluchea presentes en la isla de Tenerife.

de plantas taladas o desbrozadas cuando están parcialmente agostadas, a finales de verano y principios de otońo. Los rebrotes de las plantas alertan sobre la necesidad de ejecutar revisiones periódicas en las localidades objeto de manejo. En todo caso, la Administración entiende que actúa sobre $P$. ovalis, aunque, sin saberlo, por la dificultad en la identificación, es probable que también lo haya hecho sobre $P$. dioscoridis si se atiende a la distribución del material muestreado. 


\section{DISCUSIÓN}

La introducción de especies exóticas invasoras por el hombre puede ser accidental, a través del cultivo de sus semillas (Mack et al. 2000) o plantas, o intencionada, al emplear especies utilizadas en silvicultura, acuicultura y horticultura (Bayón \& Vilà, 2019). En la isla de Tenerife, parece que en el caso de P. ovalis, su introducción se podría deber a motivos ornamentales (Padrón-Mederos et al. 2009; Verloove \& Reyes-Betancort 2011), probablemente por su plantación en el campo de Golf de Adeje (Verloove \& Reyes-Betancort 2011). Además, su presencia en otras de estas instalaciones (campo de golf Los Palos, Golf de San Miguel de Abona, Amarilla Golf, San Andrés Golf del Sur, Golf Costa Adeje y Abama Golf) hace pensar que los propágulos también podrían haber llegado en la arena que se emplea como sustrato base, ya que al parecer en los últimos ańos las arenas utilizadas en la isla de Tenerife se traen desde el noroeste de África, en concreto, desde El Aaiún (A. Aguiar, com. pers.).

Su propagación y expansión debe atribuirse a la elevada producción de semillas (aquenios vilanados) que se dispersan con facilidad por anemocoria (Barone $\&$ Hernández 2014; Martín-Osorio \& Wildpret 2014). Las semillas que caen al suelo quedan sujetas al transporte por la acción del agua de lluvia y de la escorrentía superficial colonizando zonas próximas, o incluso avanzar a través de barrancos y/u obras de drenaje, como pudiera tratarse de los ejemplares localizados en la desembocadura del barranco encauzado de la Reserva Ambiental San Blas, conducciones soterradas con desagüe en la playa de La Caleta, obra de drenaje en el barranco del Golf del Sur o el barranco anexo a la Finca Abama.

Stiles (2000) apunta que las aves son uno de los mejores vectores de dispersión de propágulos. En especial, las aves acuáticas suponen una importante vía de transporte de semillas (Figuerola \& Green 2002). Los ecosistemas hidrofíticos en los que crecen estas dos especies (principalmente en bordes e interior de charcas, estanques y presas) posibilitan la visita regular de fauna ornitológica que favorecería la dispersión de sus semillas. Además, el uso como recurso alimenticio y para la construcción de nidos por parte de paseriformes (R. Jorge, com. pers.) también corrobora que la avifauna es uno de los vehículos de su dispersión.

Volviendo a los campos de golf del sur de la isla, la propagación de $P$. ovalis y $P$. dioscoridis entre distintas instalaciones puede obedecer al trasiego de jugadores, siendo la dispersión a través de los zapatos y/o la indumentaria, tal como se informa por parte del Greenkeeper del Real Club de Golf de Tenerife.

Las carreteras promueven la dispersión de especies exóticas al alterar los hábitats y proveer corredores para el movimiento (Trombulak \& Frissell 2000). En este sentido, la presencia de ejemplares de alguna de estas dos especies del género en los márgenes de las carreteras del sureste y suroeste de la isla hace presagiar que estas especies ampliarán su área de distribución alcanzando el norte de la isla, máxime cuando se han iniciado las obras para dar continuidad al corredor principal en el noroeste y cerrar el circuito de carreteras rápidas de la isla.

Por último, el carácter alelopático identificado en especies del género $\mathrm{Plu}$ chea, relacionado con la presencia de compuestos fenólicos en hojas y raíces de, por 

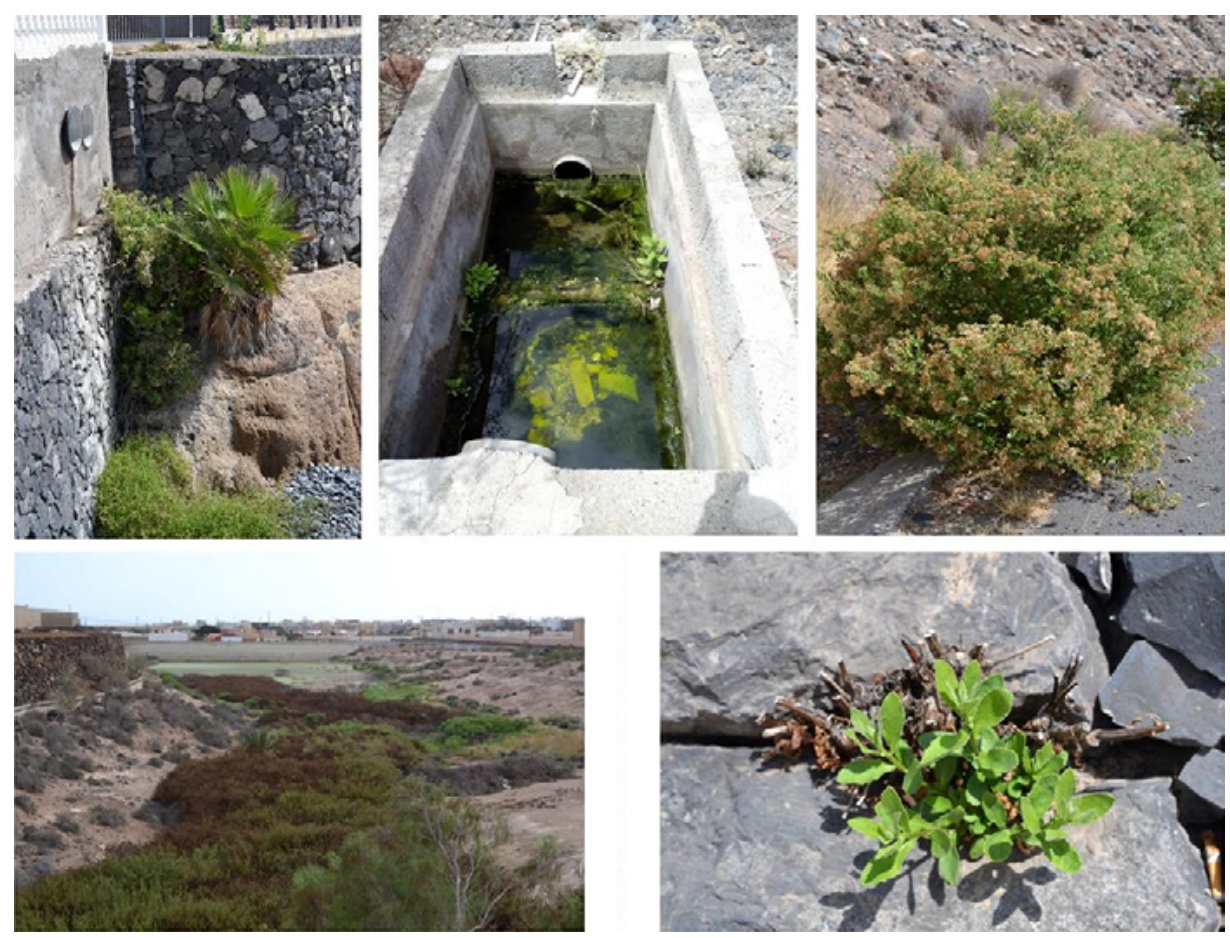

Figura 7. Explosión de las especies del género Pluchea en ámbitos de influencia antrópica, de izquierda a derecha y de arriba abajo: playa La Caleta, interior de una tanquilla de riego con agua, margen de carretera, charca de Las Galletas o de Don Casiano (Arona, Tenerife) y en las oquedades de un muro.

ejemplo, P. lanceolata (Dakshini 1994) y P. dioscoridis (Fahmy et al. 2012), favorece su expansión en el entorno más próximo al controlar la germinación y el crecimiento de otras especies vegetales. El monopolio de $P$. ovalis y $P$. dioscoridis en la charca de Las Galletas o de Don Casiano y en la charca de la Reserva Ambiental San Blas podrían explicarse, en parte, por esta propiedad.

Estas especies han encontrado ámbitos propicios para su asentamiento y expansión en la isla de Tenerife. Principalmente en los de fuerte influencia humana que, en ocasiones, están asociados a aportes adicionales de agua, como bordes de charcas y presas, atarjeas, jardines con riego por goteo, márgenes de carretera, fondos de barrancos, bordes de invernaderos, muros de piedra, solares y otros lugares con humedad edáfica (ver figura 7). 


\section{CONCLUSIONES}

La actual distribución de $P$. ovalis y $P$. dioscoridis supone un incremento espacial con respecto a los datos conocidos hasta el momento. Además, su llegada a los espacios naturales protegidos de la Red Canaria y de Natura 2000 pone en riesgo el equilibrio de sus hábitats y especies, como podría llegar a suceder con el amenazado lagarto gigante de Tenerife (Gallotia intermedia Hernández, Nogales \& Martín, 2000), en la fuente de Borrallos, dentro del Parque Rural de Teno y ZEC homónimo (J.M.R. Carmona, com. pers.), lo que podría poner en peligro los recursos alimenticios de esta especie protegida, por competencia de ambas especies de Pluchea con plantas autóctonas de la zona por el espacio.

Para la contención y control de la expansión de estas especies es necesario que se dé continuidad a los trabajos que, desde las distintas escalas de la Administración, se llevan ejecutando desde 2017, por otra parte es necesario contar con sectores del ámbito turístico (hoteles y campos de golf) y comercial para evitar su venta. Es importante la formación del personal con competencia en gestión de parques y jardines en el reconocimiento de las especies y en los métodos de control, tanto municipales como de establecimientos turísticos y de viveros.

Las características de estas especies exóticas como plantas potencialmente invasoras (elevada producción de semillas, rápida expansión y múltiples vías de dispersión) pueden facilitar el salto a cualquier otra isla. Resulta, por lo tanto, primordial el traslado de la información al resto de cabildos y ayuntamientos del archipiélago, en particular a los de las islas de La Gomera, La Palma y El Hierro, con los que se mantiene conexión vía línea marítima desde el sur de la isla de Tenerife a través del puerto de Los Cristianos.

La inclusión de estas especies en el vigente Catálogo español de especies exóticas invasoras -y en el futuro Catálogo regional- desplegaría sus efectos jurídicos, favoreciendo, entre otras cuestiones, que las políticas medioambientales de control y erradicación se focalicen sobre estos taxones, dedicando recursos para acotar y frenar la expansión, evitar la plantación y/o mantenimiento en zonas públicas, instalaciones hoteleras, campos de golf y jardines en general y, de paso, abordar aspectos como la educación ambiental, herramienta imprescindible para el control y gestión de estas especies.

\section{AGRADECIMIENTOS}

Al Cabildo Insular de Tenerife, por la sesión de los datos del Proyecto de Control de Flora Exótica Invasora de Tenerife; a Alejandro Martín y Moisés Dorta, personal del proyecto REDEXOS, por aportar su experiencia con estas especies; a los naturalistas Rubén Barone y Fabián Hernández, por ceder sus datos de distribución de Pluchea ovalis; a Ricardo Mesa Coello, por la revisión del texto y sus aportaciones; a Agustín Aguiar y Rayco Jorge, por sus comunicaciones personales; a Jorge Alfredo Reyes-Betancort, director del Jardín de Aclimatación de La Orotava, por su asesoramiento taxonómico; y a Javier Martín-Carbajal, David Pérez Padilla y Juan 
Manuel R. Carmona, por sus datos de campo. Asimismo, a los diferentes directores de los campos de golf visitados: Los Palos, Amarilla Golf, San Andrés Golf del Sur, Golf Costa Adeje, Abama Golf, Buenavista Golf y Real Club de Golf de Tenerife, por su colaboración en los trabajos de prospección; a Manuel Padilla, por recolectar muestras de las plantas-objetivo; a Elizabeth Ojeda-Land y Juan Luis Rodríguez Luengo, técnicos del Servicio de Biodiversidad del Gobierno de Canarias, por sus comentarios sobre las especies, y a los biólogos Pilar Bello Bello y Jesús Brito, por su colaboración en el desarrollo de este trabajo.

\section{CONTRIBUCIÓN DE LOS AUTORES}

Conceptualización: BFT.

Metodología y trabajo de campo: BFT, ARQ, MLRN.

Análisis de datos: BFT, ARQ, MLRN.

Preparación del escrito original: MLRN.

Corrección y edición del escrito definitivo: BFT, ARQ, MLRN, RMC.

Recibido: diciembre de 2019, aceptado: agosto de 2020 


\section{REFERENCIAS}

Banco del Inventario Natural de Canarias (https://www.biodiversidadcanarias.es).

Barone Tosco, R. y Hernández Romero, F. 2014. La planta Pluchea ovalis invade el sur de Tenerife. Quercus 339: 42-43.

BAYÓn. Á. y VILÀ, M. 2019. Horizon scanning to identify invasion risk of ornamental plants marketed in Spain. NeoBiota 52: 47-86.

Catálogo español de especies exóticas invasoras -Real Decreto 630/2013, de 2 de agosto, publicado en el BOE núm. 185, de 3 de agosto de 2013-.

Chevalier, A. 1935. Les Iles du Cap Vert. Géographie, biogéographie, agriculture. Flore de l'Archipel. Revue de botanique appliquée et d'agriculture coloniale 15 année, n. ${ }^{\circ}$ 170-171: 733-1090.

Dakshini, K.M.M. 1994. Allelopathic potential of the phenolics from the roots of Pluchea lanceolata. Physiologia Plantarum 92(4): 571-576.

Elejabeitia Velu, Y. y Delgado Bello, J.A. 2018. Priorización actuaciones con especies exóticas invasoras en Tenerife, aprendiendo de la experiencia. Comunicación técnica presentada en: Congreso Nacional del Medio Ambiente CONAMA 2018. (http://www.conama2018.org).

Fahmy, G.M., Al-Sawaf, N.A., Turki, H. y Ali, H.I. 2012. Allelopathic potential of Pluchea dioscoridis (L.) DC. J. Appl. Sci. Res. 8(7): 3129-3142.

Figuerola, J. y Green, A.J. 2002. How frequent is external transport of seeds and invertebrate eggs by waterbirds? A study in Doñana, SW Spain. Archiv fur Hydrobiologie 155(4): 557-565.

Global Biodiversity Information Facility (GBIF-https://www.gbif.org).

Global Register of Invasive Species (GRIIS-https://www.griis.org).

Plant of the World online. Royal Botanic Gardens, Kew (http://www.plantsoftheworldonline.org).

King-Jones, S. 1999. Studies in the Compositae of the Arabian Peninsula and Socotra-4. The Arabian species of Pluchea (Compositae, Plucheeae). Willdenowia 29: 203-220.

KIng-Jones, S. 2001. Revision of Pluchea Cass. (Compositae, Plucheeae) in the Old World. Englera 23: 3-136.

Mack, R.N., Simberloff, D., Lonsdale, W.M., Evans, H., Clout, M. y Bazzaz, F.A. 2000. Biotic Invasions: Causes, epidemiology, global consequences and control. Ecological Applications 10(3): 689-710.

Martín-Osorio, V.E. y Wildpret, W. 2014. Pluchea dioscoridis (L.) DC (Asteraceae) nueva especie introducida y naturalizada en Tenerife, Islas Canarias. Vieraea 42: 323-326.

Oliver i Martínez-Fornés, X., Casals Tortras, P., Fanlo Grasa, E. y García Pausas, J. 2007. Contribuciones a la flora vascular de Marruecos. 13. Pluchea ovalis (Pers.) DC., nueva cita en el Valle del Sous (Marruecos). Acta Botanica Malacitana 32:301-304.

Padrón-Mederos, M.A., Guma, I.R., Santos-Guerra, A. y Reyes-Betancort, J.A. 2009. Apuntes florísticos y taxonómicos para la flora de las islas canarias. Acta Botanica Malacitana 34: 242-251.

Qhiser, M. у Abid, R. 2003. En: Flora of Pakistan. Asteraceae (II), vol. 210. [Internet]. Cita Nov 2019. Extraído de http://www.efloras.org. 
Shaltout, K.H. y Slima, D.F. 2007. The biology of Egyptian woody perennials 3. Pluchea dioscoridis (L.) DC. Ass. Univ. Bull. Environment Res. 10(1): 85-103.

Simpson, N.D. 1932. A report on the Weed Flora of the Irrigation Channels in Egypt. Ministry of Public Works, Government Press, Cairo. 28 p.

Sistema de Información Territorial de Canarias-IDECanarias (https://www.idecanarias.es).

Stiles, F.G. 2000. «Animals as seed dispersers», en Fenner, M. (ed.). Seeds: The Ecology of Regeneration in Plant Communities. p. 111-124. CABI Publishing.

Trombulak, S.C. y Frissell, C.A. 2000. Review of ecological effects of roads on terrestrial and aquatic communities. Conservation Biology 14(1): 18-30.

Verloove, F. y Reyes-Betancort, J.A. 2011. Additions to the flora of Tenerife (Canary Islands, Spain). Collectanea Botanica 30: 63-78. 


\section{ANEXO I}

Exsiccata: Pluchea ovalis (Pers.) DC. Tenerife:

Charca San Blas, San Miguel de Abona, 03.X.2019, (UTM-WGS 84-: 342443/3102245, a 43 m de altitud), (Leg. B. Farińa y A. Rivero), (ORT47097 + duplic.); Ibid., 17.XI.2019, (UTM-WGS 84-: 342443/3102245, a 43 m de altitud), (Leg. B. Farińa y M.L. Rodríguez Navarro), (ORT47098); carretera San Isidro-Cueva Hermano Pedro, Granadilla de Abona, 03.X.2019, (UTM-WGS 84-: 347119/3105065, a 113 m de altitud), (Leg. B. Farińa y A. Rivero), (ORT47099 + duplic.); carretera Fañabé-La Caleta, Adeje, 06.X.2019, (UTM-WGS 84-: 329718/3109955, a $121 \mathrm{~m}$ de altitud), (Leg. B. Fariña, A. Rivero y M.L. Rodríguez Navarro), (ORT47102); bco. del Agua, carretera Fañabé-La Caleta, Adeje, 06.X.2019, (UTM-WGS 84-: 329697/3110322, a $117 \mathrm{~m}$ de altitud), (ejusd.), (ORT47103); Golf Los Palos, Arona, 11.X.2019, (UTM-WGS 84-: 334809/3102552, a $65 \mathrm{~m}$ de altitud), (ejusd.), (ORT47107); Los Gigantes, Santiago del Teide, 14.X.2019, (UTM-WGS 84-: 319699/3127158, a 20 m de altitud), (Leg. M. Padilla), (ORT47109 + duplic.); cauce de Masca, Buenavista, 23.X.2019, (UTM-WGS 84-: 317504/3130855, a $48 \mathrm{~m}$ de altitud), (ejusd.), (ORT47111); hotel Abama, Guía de Isora, 31.X.2019, (UTM-WGS 84-: 322962/3117355, a 51 m de altitud), (Leg. M.L. Rodríguez Navarro y A. Rivero), (ORT47112).

\section{Pluchea dioscoridis (L.) DC. Tenerife:}

Márgenes del Campo de Golf de San Miguel de Abona, 06.X.2019, (UTM-WGS 84-: 340730/3100865, a 25 m de altitud), (Leg. B. Fariña, A. Rivero y M.L. Rodríguez Navarro), (ORT47100); Casa Club Amarilla Golf, San Miguel de Abona, 06.X.2019, (UTM-WGS 84-: 340965/3101747, a 58 m de altitud), (ejusd.), (ORT47101 + duplic.); trasera Universidad de Verano de Adeje, 06.X.2019, (UTM-WGS 84-: 330496/3111975, a $286 \mathrm{~m}$ de altitud), (ejusd.), (ORT47104); solar en el entorno de la Universidad de Verano de Adeje, 06.X.2019, (UTM-WGS 84-: 330550/3112038, a $295 \mathrm{~m}$ de altitud), (ejusd.) (ORT47105); paseo de Los Pinalitos-Caleta de Adeje, 06.X.2019, (UTM-WGS 84-: 327315/3109817, a $28 \mathrm{~m}$ de altitud), (ejusd.) (ORT47106); charca de Las Galletas, Arona, 11.X.2019, (UTM-WGS 84-: 336203/3101026, a 43 m de altitud), (ejusd.), (ORT47108); Chimiche, Granadilla de Abona, en el interior de una represa actualmente seca, 18.X.2019, (UTM-WGS 84-: 352117/3110325, a 155 m de altitud), (Leg. A. Rivero y M.L. Rodríguez Navarro), (ORT47110); jardines de la Urbanización Ocean Boulevard en Amarilla Golf, 17.XI.2019, (UTM-WGS 84-: 341639/3101375, a $49 \mathrm{~m}$ de altitud), (Leg. M.L. Rodríguez Navarro y B. Fariña), (ORT47113); desembocadura del barranco encauzado de la Reserva Ambiental San Blas, 17.XI.2019, (UTM-WGS 84-: 342779/3101375, a 5 m de altitud), (Leg. B. Fariña y M.L. Rodríguez Navarro), (ORT47114). 
